$\xi$

\title{
Multicasting technique for path establishment in vehicular ad hoc networks
}

\author{
Sukhpreet Kaur $^{1 *}$, Er.Sharad ${ }^{2}$ \\ ${ }^{1}$ M. tech Scholar Department of Computer Science and Engineering, Chandigarh University, Mohali , India \\ ${ }^{2}$ Assistant Professor Department of Computer Science and Engineering ,Chandigarh University , Mohali , India \\ *Corresponding author E-mail: preetsahota525@gmail.com
}

\begin{abstract}
The vehicular adhoc network is the decentralized type of network in which vehicles can move from one location to another. In the network two type of communication is possible which are vehicle to vehicle and vehicle to infrastructure. In the vehicle to vehicle type of path establishment is the major issue of the network. In this research work, multicasting routing technique is proposed for the path establishment from source to destination. The proposed technique is implemented in NS2 and simulation results shows improvement in network delay for path establishment
\end{abstract}

Keywords: Vanets; Multicasting; LAR; AODV

\section{Introduction}

A wireless network that is self-organized and autonomous is nature is known as Vehicular ad hoc network. It provides both, vehicle to vehicle as well as vehicle to roadside communication within the network. In order to exchange information amongst each other, the nodes act as both clients and servers. The utilization of wireless communication in order to increase the road safety is the prior objective of VANETs [1]. The vehicles act as sensors and any abnormality or hazardous conditions identified within the regions are informed to other vehicles. Due to their quick changes in topology, these networks are very similar to the ad hoc networks. Thus, there is a need to introduce routing protocols within them. There are several safety applications in which the VANETs are deployed [2]. Due to this, the drivers can be informed regarding any congestion, businesses and services within the surrounding regions of a vehicle. There are several unique propertiesidentified within VNAETs. There is random change is topology due to the higher mobility of nodes. Due to its unpredictable dynamic topology and frequent disconnections, VANET is one of the most challenging areas. Within the vehicular system, safety and security are ensured with the help of this network. As mentioned, it

Provides both Vehicle-to-vehicle and Vehicle-to-Infrastructure communications. Within both of these networks, the information is generated and distributed through vehicular communication [3]. It is very difficult to design the routing protocols in VANETs due to the highly dynamic topology they include. There are several routing protocols introduced within this network. These routing protocols are broadly classified on the basis of their properties and tasks. Amongst these classification, the topology based routing protocols are introduced in which the packet forwarding is performed with the help of including information related to links. The protocols included under this category are further classified as being proactive and reactive [4]. The maintenance of routing information in the background irrespective of the communication requests being generated is defined as proactive routing. Further, the routing in which the route is open only when communication is required amongst the vehicles is known as reactive type of routing in VANET. A class routing algorithm is available within the position based routing. In order to choose the next forwarding hops, the geographical positioning information is utilized here [5]. The transmission of packets to the destination in least possible time is the major aim of Position Based Greedy V2V protocols which are also known as min delay routing protocols. On the basis of the fact that the city street forms a natural planner graph, the Greedy Perimeter Coordinator Routing (GPCR) is developed and there is no need to external static streets map here. The protocols that identify the route towards the destination and maintain the cache of successful route amongst several source and destination pairs are known as Connectivity Aware Routing Protocols (CAR) protocols. A series of diagonal intersections is generated amongst the source and destination vehicle through Diagonal-IntersectionBased Routing Protocol (DIR) protocol [6]. The location of node in order to carry a message is not difficult in urban regions. However, at night in the cities or in rural areas, the establishment of end to end route is tough since there are fewer vehicles running on road and to solve such issues Delay Tolerant Protocols are applied. The algorithm that is introduced for space VANET conditions is called Motion Vector Routing Algorithm (MOVE). The vehicles act as mobile routers that have intermittent connectivity amongst the vehicles here. When a node enters within a broadcast range, the packets that are carried by the vehicles in sparse conditions are forwarded with the help of carry and forward strategy within Vehicle Assisted Data Delivery (VADD) protocol. The minimization of message delivery delay within the sparse networks is done through Static Node Assisted Adaptive

Routing Protocol (SADV) protocol [7]. The changing traffic density is adapted here such that the amount of time required to deliver a message can be measured by each node in this protocol. The performance of routing within the clusters can be done using cluster based routing. The clusters are generated by identification of group of similar nodes and cluster head is chosen amongst them through which the packets are broadcasted amongst the cluster. In 
order to share several types of information amongst vehicles regarding weather, traffic, road conditions and advertising and announcing them, broadcast routing is utilized [8]. A location based multicast routing in which the packet is delivered from source to all other nodes present within a defined geographical region called Zone of Relevance (ZOR), is known as Geocast routing in VANETs.

\section{Literature review}

Kais Elmurtadi Suleiman, et.al (2017) proposed a novel routing protocol for highway applications on the basis of V2I routing, $\mathrm{V} 2 \mathrm{~V}$ direct routing as well as $\mathrm{V} 2 \mathrm{~V}$ delay-tolerant routing [9]. Adaptability and incentive-compatibility are the two properties of this protocol. A credit-based exchanges system is implemented here and the fixed network operator is the administrator. The credits that earned during $\mathrm{V} 2 \mathrm{~V}$ direct routing and $\mathrm{V} 2 \mathrm{~V}$ delay-tolerant routing are utilized in order to allocate V2I routing bandwidths. By using a game theoretic analysis, the incentive compatibility of proposed protocol is shown. Several evaluations are performed in order to evaluate the performance of proposed protocol in MATLAB simulator and the results achieved shown that in comparison to other routing protocols, the proposed protocol is more efficient.

Mahabaleshwar S. Kakkasageri, et.al (2017) proposed in this paper a multi-agent based routing mechanism for VANETs [10]. By ensuring minimum communication delay, overhead as well as complexity, the data routing is to be performed. There are static as well as mobile agents present within the multi-agents base scheme. Simulations are performed which shown that in terms of parameters such as packet delivery ratio, bandwidth utilization, and route identification delay, the proposed routing mechanism performs better than other. Muhammad Tahir Abbas, et.al (2017) proposed in this paper a probabilistic model that utilized highway mobility model for Two-Level hierarchical routing protocol in order to estimate the path duration [11]. There are several analytical models proposed for various protocols in VANETs. Thus, in order to solve such issues, the road aware routing protocols are developed through mathematical model and the performance is evaluated.

Chen Chen, et.al (2016) proposed a new routing protocol such that the optimal route can be chosen as per the information of paths [12]. The artificial spiders are utilized here in order to propose the protocol named as Artificial Spider-web based Geographic Routing (ASGR) protocol. A robust route towards the destination is identified by including route connectivity, path delay as well as total number of hops in ASGR through the construction of spiderweb and path tree. Simulation experiments were conducted and it was seen that in terms of packet delivery ration and end to end delay, ASGR performed better in comparison to GyTAR and STAR.

Weiwei Dong, et.al (2016) proposed a Geographic Opportunisticrouting protocol on the basis of Link state and

forwarding quality present within the nodes [13]. Initially a

hybrid filtering and prioritizing approach is put forth and a

candidate nodes sent that sends node within LFGOR is determined. This helps in determining the link state as well as forwarding quality of node along with the position information. Thus, the reliability of transmission is enhanced along with the minimization of end-to-end delay that is caused due to timer-based scheduling mode present within many normalopportunistic routing protocols due to implementation of LF-GOR. Packetdelivery rate, throughput and the acceptable delay are found to be better in the proposed protocol in comparison to existing protocols as per the simulation.

\section{Proposed model}

In order to enhance the performance of protocol, the lifetime of a route is to be increased for which the stability of route present in between source and destination is improved here.

The nodes that participate within the route request and travel in the similar direction of movement are selected here. This is done due to the fact that the nodes that move in opposite directions generate the route that can break easily in comparison to the nodes moving in similar direction. Thus, the selection of direction of movement is an important factor to be considered.

1) Direction of movement:

The area where $\mathrm{D}$ is located can be estimated by $\mathrm{S}$ in case when a route request is required to be sent from node $S$ to node $D$ at $t 1$ time. However, the direction of movement for the destination node is not known to $\mathrm{S}$ which must be known. The nodes that move in the similar direction $\mathrm{D}$ are no possible to be known. However, the direction of movement of $\mathrm{S}$ in time $\mathrm{t} 1$ is known here which means that the movements of nodes that go in same direction of $S$ are known. A route request is proposed to be sent to the nodes that are moving in similar direction to that of a source in order to enhance the stability of path amongst source and destination on the highway scenario.

The direction of motion in comparison to $\mathrm{S}$ is checked when a route request is received by a node. The route request is

Retransmitted in case it moves in the similar direction to that of $\mathrm{S}$ or the route request is eliminated otherwise. Upon the constraints of LAR scheme 1 (LAR1), this proposition is added.

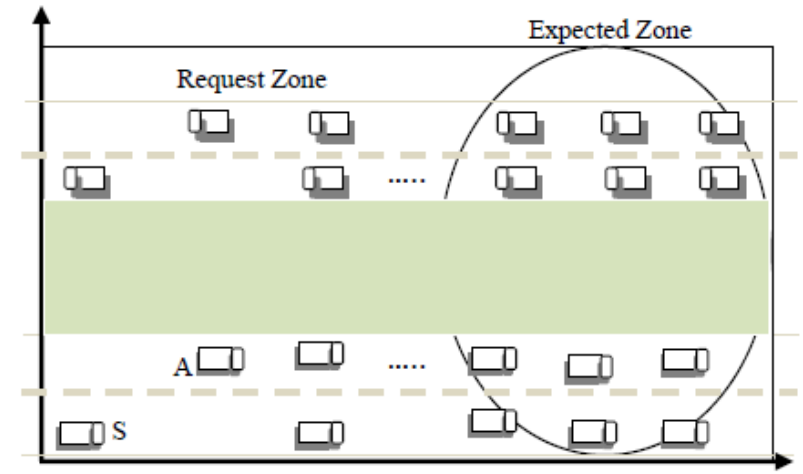

Fig. 1: Bi-Directional Highway Model.

Figure 1 represents an example scenario here. The route request node I is forwarded nu node A such that the route request can be forwarded due to the fact that its direction of movement is similar to that of S. The route request is deleted in case if 'I' does not have the similar direction to that of $\mathrm{S}$.

2) The neighbor to stay longest time:

The transmission of route request message to the vehicle that has maximum time within the coverage area where the vehicle is transmitting is proposed here. The process of transmission of route request to all the vehicles that are traveling within the similar direction of motion of source $\mathrm{S}$ can be eliminated here.

The time for which a vehicle has to remain in the coverage area such that the vehicle is in the half-circle of the communication range within the side that is near to destination (D), is computed, as shown in figure 2 . The receiver vehicle of the route request message is chosen on the basis of the vehicle that has longest time. Till the message reaches the destination within the constraints of LAR1 protocol, the procedure keeps iterating. In order to compute the time of each neighbor, four different cases are presented here. The transmission of each vehicle is assumed to be transmitted to the neighbors here. The position of vehicle $\mathrm{A}$ at time $\mathrm{t} 0$ is $(X A$, and $V A$ is its speed. The speed of neighboring vehicle $\mathrm{I}$ is $V I$ and it is located at position $(, Y I)$. The coverage area of vehicle $\mathrm{A}$ is left by vehicle I at time $\mathrm{t} 1$. Thus, $\mathrm{t} 0=\mathrm{t} 1-\mathrm{t} 0$ is the time for which the vehicle stays within the coverage area. For instance, amongst A and $\mathrm{I}, \mathrm{d}$ and $\mathrm{h}$ are the distances taken respectively. The distances 
are taken at time $\mathrm{t} 0$ on abscissa and ordinate axis. Then, at time $\mathrm{t} 1$, the distance between A and I on abscissa axis is denoted as ' $a$ '. The distance traveled by vehicle $I$ at time $t 1$ is denoted as ' $x$ '. Further, during time $t=t 1-t 0$, the speed of vehicle is assumed to be constant. However, the speeds of each of the vehicles are different.

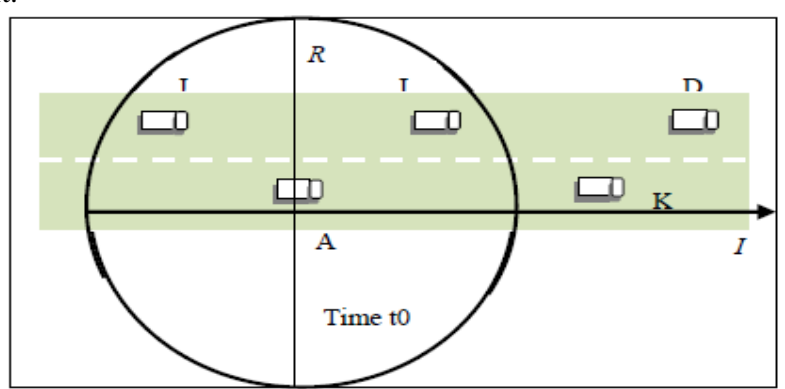

Fig. 2: Half-Circle of the Communication Range in the Side Closing to the Destination.

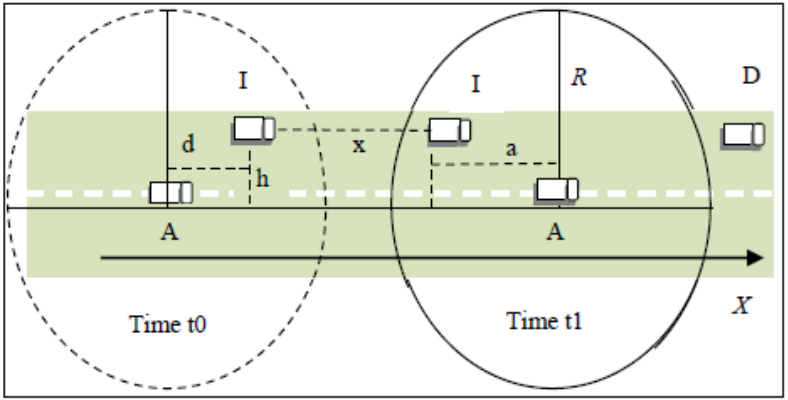

Fig. 3: D Is in the Direction of Movement of S and VA > VI.

First case: At time t0, in comparison to I, the speed of A is Strictly higher and in the direction of S, the destination moves. As shown in figure 3 , the distances travelled by A and I at

Time $\mathrm{t} 1$ can be calculated as:

$$
d+x+a=t * V_{A}
$$

Thus,

$$
\begin{aligned}
& x=\frac{v_{1}}{V_{A}-V_{t}} *(d+a) \\
& d=\left|X_{I}-X_{A}\right| \\
& a=\sqrt{R^{2}-\left(Y_{I}-Y_{A}\right)^{2}}
\end{aligned}
$$

Hence,

$$
t=\frac{\left|x_{I}-x_{A}\right|}{V_{A}-V_{I}}+\frac{\sqrt{R^{2}-\left(Y_{X}-Y_{A}\right)^{2}}}{V_{A}-V_{I}}
$$

\section{Experiments and results}

On the basis of mobility model that is selected such that accurate simulation results can be achieved, the performance studies of VANET protocols are dependent. It is important to include realistic vehicular mobility scenarios in order to enhance the performance of LAR protocol. During the simulation time in a simulated area, the vehicular motion is defined by mobility model. The real behavior of the vehicular traffic is reflected with the help of this model. Thus, the IDMLC pattern which is generated per VANET mobility simulation (VanetMobiSim) is utilized here. The movement pattern for a highway is generated with the help of this pattern. In order to perform, NS-2 simulator is utilized here. Within the area of $5000 \mathrm{~m} \times 100 \mathrm{~m}$, the vehicles are deployed. In order to reach to the required velocity, the vehicles move and accelerate. If there are multiple lanes present in a road, the vehicles that are moving with each other try to overtake others. In order to avoid shock, the vehicle however, decelerates if it cannot overtake others. A state of traffic sign is acquired in case a vehicle is approaching at a dead end of road. The vehicle decelerates and stops in case the stop sign is found. In order to intersect, it slightly reduces its speed. The IEEE 802.11 MAC layer is utilized in order to help the vehicles to communicate with each other. On the basis of different node densities, the simulations are repeated for vehicles. Within the range of $22 \mathrm{~m} / \mathrm{s}$ and $27 \mathrm{~m} / \mathrm{s}$, the average speed of vehicles is found within a highway that includes multiple lanes. Through the exchange of information, the vehicles get the set of one hop vehicles. With the help of HELLO messages, the information is achieved and each HELLO period that was set of $1 \mathrm{~s}$ is now updated. $250 \mathrm{~m}$ is the transmission range provided.

Table 1: Mobility Model Parameters

\begin{tabular}{|c|c|}
\hline Parameter & Value \\
\hline Number of lanes & 2 \\
\hline Motion mode1 & IDM_LC \\
\hline Vehicle Length & $0.5 \mathrm{~m} / \mathrm{s} 2$ \\
\hline $\begin{array}{c}\text { The comfortable deceleration } \\
\text { of movement }\end{array}$ & $0.1 \mathrm{~s}$ \\
\hline $\begin{array}{c}\text { The step for recalculating } \\
\text { movement parameters }\end{array}$ & \\
\hline
\end{tabular}

\section{Packetloss Comparision}

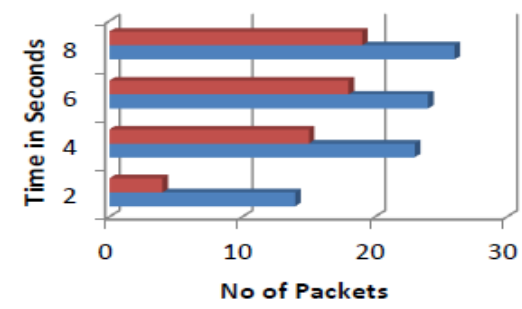

E-AODV

AODV

Fig 4: Packet Loss Comparison.

As shown in the figure 4, packetloss criteria are used to compare the old as well as the new proposed technique. The packetloss is found to be less in the new proposed technique than the already existing technique.

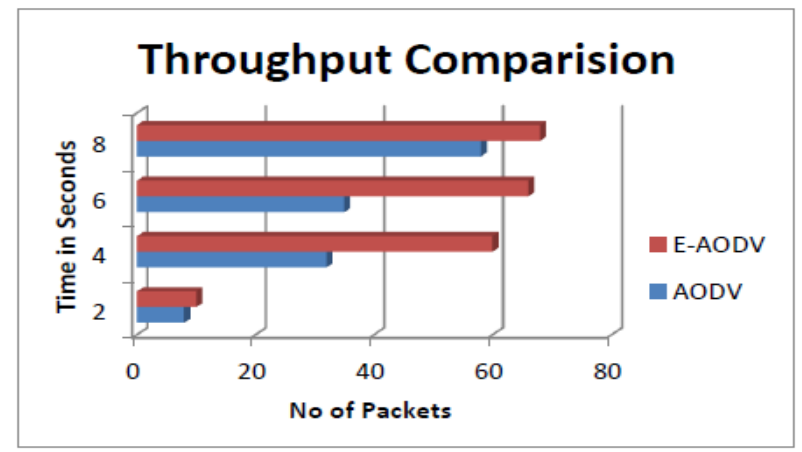

Fig 4: Throughput Comparison

As shown in figure 4, for establishing a path the broadcasting technique is used. In addition, the multicasting technique is applied by the proposed algorithm in the network. Due to this reason, the throughput of the network is increased.

\section{Conclusion}

In this paper, it is conclude that path establishment is the major issue of vehicle adhoc network due to high mobility and dynamic nature of the network. In this research work, 
multicasting technique is proposed for the path establishment from source to destination. The proposed technique is based on zonal routing which is divided into expected and predicted zones. The simulation results shows that proposed technique performs well in terms of packetloss and throughput.

\section{References}

[1] S. Zeadally, R. Hunt, Y. S. Chen, A. Irwin, and A. Hassan,"Vehicular ad hoc networks (VANETS): Status,

Results, andchallenges," Telecommunication Systems, vol. 50,pp. 217-241, 2012.

[2] C. Chen, Y. Jin, Q. Pei, and N. Zhang, "A connectivityawareintersection- based routing in VANETs," EURASIP Journal onWireless Communications and Networking, vol. 2014, pp. 1-16, 2014.

[3] J. J. Chang, Y. H. Li, W. Liao, and C. Chang, "Intersectionbasedrouting for urban vehicular communications with trafficlightconsiderations," Wireless Communications, IEEE, vol. 19, pp. 82-88,2012.

[4] M. Jerbi, S. M. Senouci, R. Meraihi, and Y. Ghamri- Doudane, "Animproved vehicular ad hoc routing protocol for city environments," inProc. IEEE International Conference on Communications, 2007, pp.3972-3979.

[5] "COMeSafety-D31 European ITS Communication Architecture,"Information Society Technologies,, vol. Tech. Rep., 2008.

[6] S. Yu, B. Zhang, C. Li, and H. Mouftah, "Routing protocols for wirelesssensor networks with mobile sinks: a Survey," IEEE CommunicationsMagazine, vol. 52, no. 52, pp. 150 $157,2014$.

[7] A. B. M. Moniruzzaman and M. S. Rahman, "Analysis of topologybased routing protocols for vehicular ad-hoc network (vanet)," EprintArxiv, vol. pp, 2014.

[8] A. Trivi09o-Cabrera and S. Ca09adas-Hurtado, "Survey on opportunisticrouting in multihop wireless networks," International Journal ofCommunication Networks \& Information Se, vol. 3, no. 2, pp. 170-176, Aug. 2011.

[9] Kais Elmurtadi Suleiman, Otman Basir, "Adaptive Incentivecompatible Routingin VANETs for Highway Applications", 2017, IEEE

[10] Mahabaleshwar S. Kakkasageri, Mamata J. Sataraddi, Poornima M. Chanal and Gururaj S. Kori, "BDI Agent Based Routing Scheme in VANETs", 2017, IEEE

[11] Muhammad Tahir Abbas, Wang Cheol Song, "A Path Analysis of Two-level Hierarchical RoadAware Routing in VANETs", 2017, IEEE

[12] Chen Chen, Lei Liu, Ning Zhang, Shengda Wang, "A Bio-inspired Geographic Routing in VANETs", 2016 IEEE International Conference on Intelligent Transportation Engineering.

[13] Weiwei Dong, Changle Li and Zhifang Miao, "Joint Link State and Forwarding Quality: A NovelGeographic Opportunistic Routing in VANETs", 2016, IEEE. 